\title{
Two-photon Excited Polarization-Dependent Autofluorescence of Amyloids as a Label-Free Method of Fibril Organization Imaging
}

Patryk Obstarczyk, Maciej Lipok, Manuela Grelich-Mucha, Marek Samoć, Joanna OlesiakBańska*

Advanced Materials Engineering and Modelling Group, Wroclaw University of Science and Technology, Wybrzeże Wyspiańskiego 27, 50-370 Wroclaw, Poland

\section{Table of Contents}

1. Insulin spherulite incubation conditions and sample preparation

2. Polarization-sensitive two-photon microscopy setup

3. Fitting model theory

4. Data analysis

5. Atomic Force Microscopy imaging of separated insulin fibrils

6. Polarization-sensitive two-photon microscopy imaging of ThT-labeled spherulites

7. References

\section{Insulin spherulite incubation conditions and sample preparation:}

Bovine insulin, DPX mountant for histology and hydrochloric acid were of analytical grade and used without further purification (Sigma-Aldrich). Solutions were made by dissolving $10 \mathrm{mg}$ of bovine insulin powder into $1 \mathrm{~mL}$ of water-hydrochloric acid mixture with $\mathrm{pH}$ adjusted to 1.5 . Thioflavin T-labeled spherulites were prepared in a similar way, with an additional portion of $0.5 \mathrm{mg}$ of ThT dye. The solutions were then incubated at $70{ }^{\circ} \mathrm{C}$ for $24 \mathrm{~h}$ without stirring. For microscopy measurements wells in microscope slides were filled with $90 \mu \mathrm{L}$ of spherulites containing solution and covered with a coverslip. To avoid dehydration the samples were sealed thoroughly with DPX mountant near glass edges. 
a)

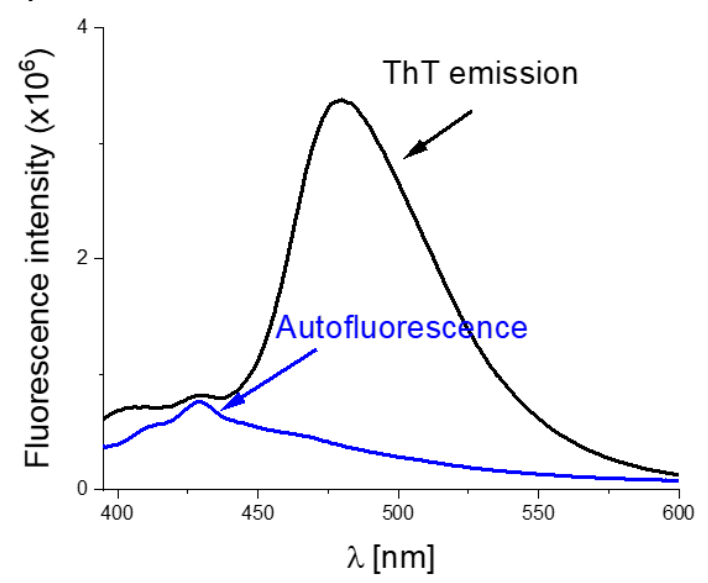

b)

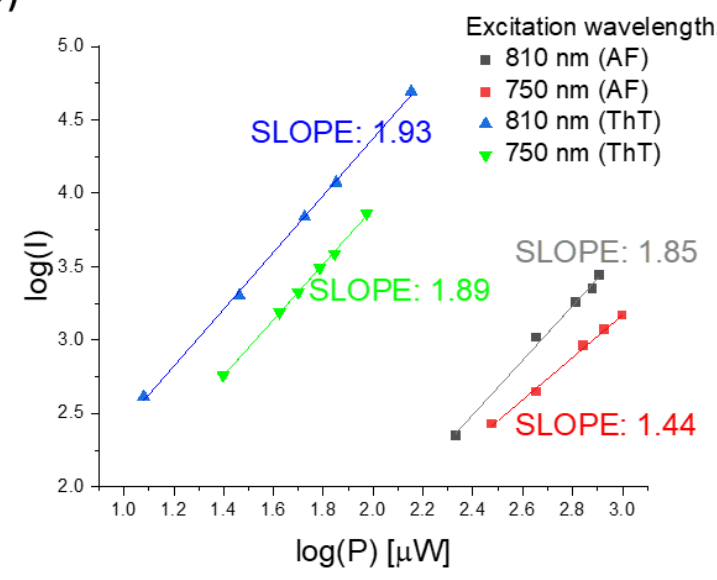

Figure S1. a) One-photon excited emission of spherulites solution labeled with ThT (excited with $375 \mathrm{~nm}$ - black line) and label-free (excited with $375 \mathrm{~nm}$ - blue line). b) 2-photon fluorescence (2PF) dependence upon excitation laser power $P$ of thioflavin labeled spherulites (blue and green triangles) and label-free spherulites (grey and red squares) excited with two wavelengths $(810 \mathrm{~nm}$ and $750 \mathrm{~nm}$, respectively). The presented dependencies are consistent with the two-photon nature of the observed processes.

\section{Polarization-sensitive two-photon microscopy setup:}

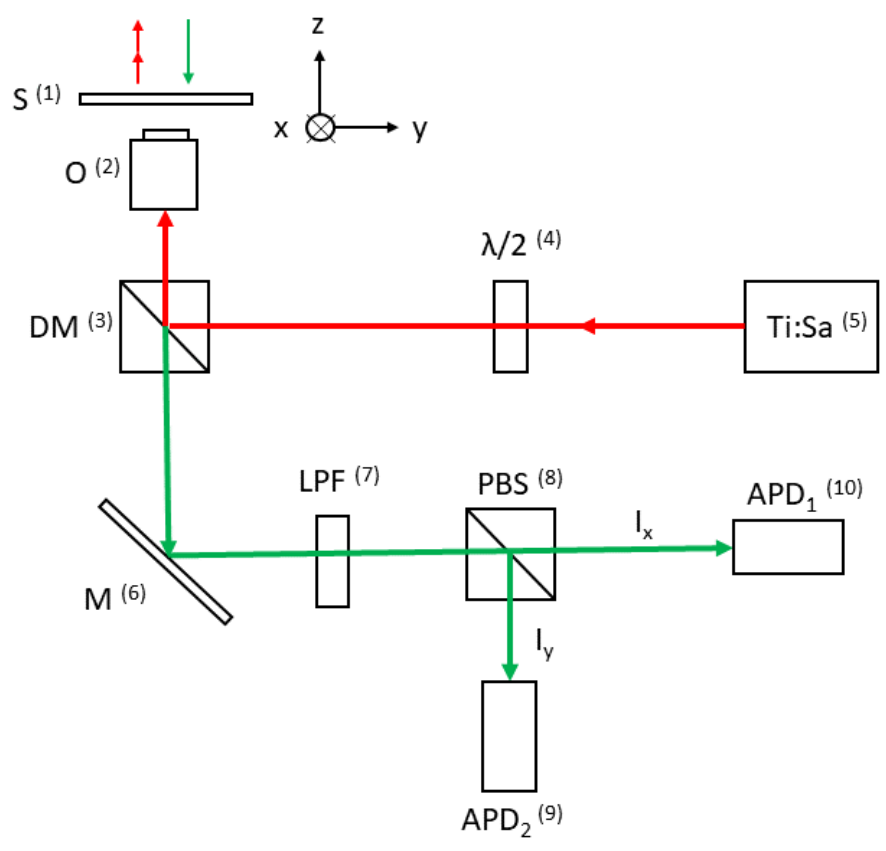

Figure S2. Two-photon microscopy setup. $S(1)$ - sample on a piezoelectric stage, $O(2)$ objective, DM (3)-dichroic mirror, $\lambda / 2$ (4) - half-wave plate, Ti:Sa (5) - laser light source, $M$ (6) - mirror, LPF (7) - $420 \mathrm{~nm}$ cut-off long-pass filter, PBS (8) - polarization beam-splitter, $A P D_{1,2}{ }^{(9,10)}$ - avalanche photodiodes detecting the $I_{y}$ and $I_{x}$ components of the emitted signal. 
Two-photon excited luminescence imaging was performed with a custom-built system consisting of a multiphoton microscope and polarization-sensitive detection path (Figure S3). The excitation light source was a fs mode-locked Ti:Sapphire laser $(\sim 100 \mathrm{fs}, 80 \mathrm{MHz}$, Chameleon, Coherent Inc.) with the incident wavelength range tunable within $\lambda=690-1080 \mathrm{~nm}$ and average power of a few $\mathrm{mWs}$. To set the polarization of the excitation light, the laser beam was passed through a half-wave plate mounted on a rotation stage. The samples were mounted on an XYZ piezoelectric scanning stage (Piezojena, TRITOR 102) and scanned to obtain images. The polarized laser beam was reflected by a dichroic mirror and focused on the sample using a high-numerical aperture objective (Nikon Plan Apo Oil Immersion 100x/1.4 NA). The backward emitted signal (epifluorescence mode) was collected by the same objective and split by a polarizing beam-splitter into two orthogonally polarized beams $\left(\mathrm{I}_{\mathrm{x}}\right.$ and $\left.\mathrm{I}_{\mathrm{y}}\right)$ to be detected by two photon-counting avalanche photodiodes (IDQ id100). In order to exclude the potential influence of SHG on the collected emission signal $420 \mathrm{~nm}$ long-pass filters were applied. The two-photon excited emission spectra were measured with a spectrograph (Shamrock 303i Andor). The signals from the samples were obtained in the range of excitation set to $750 \mathrm{~nm}$ and $810 \mathrm{~nm}$, with power of the incident laser beam set within 100-900 $\mu \mathrm{W}$ to $10-50 \mu \mathrm{W}$ for auto-fluorescence measurements and thioflavin labeled spherulites, respectively. The experimental conditions were chosen to prevent photobleaching and achieve a high signal to noise ratio.
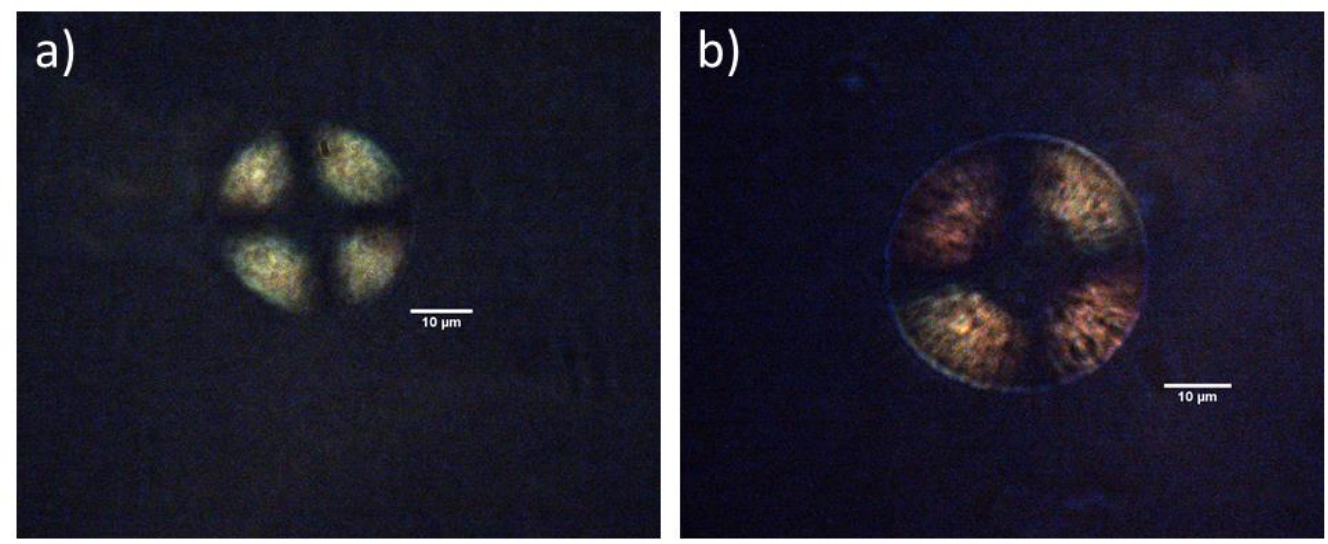

Figure S3. ThT-labeled a) and label-free b) spherulites imaged under polarized light microscope with crossed polarizers prior to two-photon imaging.

\section{Fitting model theory:}

The fitting model is based on the description of two-photon excited fluorescence intensity by S. Brasselet ${ }^{1}$.

For each molecule the measured $2 \mathrm{PEF}$ intensity $I_{I}^{2 P E F}$ is proportional to the probability of absorption $P_{a b s 2}$, depending on its transition dipole moment $\vec{\mu}_{a b s}$ and incident electric field $\vec{E}_{\omega}$. Probability of the emission $P_{e m}$ depends on molecular emission dipole $\vec{\mu}_{e m}$ and the analysis axis $\vec{\mu}_{I}$ (detection polarization):

$$
\begin{aligned}
P_{a b s 2} & \propto\left|\vec{\mu}_{a b s} \times \vec{E}_{\omega}\right|^{4} \\
P_{e m} & \propto\left|\vec{\mu}_{e m} \times \vec{\mu}_{I}\right|^{2}
\end{aligned}
$$


$2 \mathrm{PEF}$ is an incoherent process and thus could be expressed as originating from superposition of $N$ single molecules in the detected volume. The orientation distribution function used to describe the molecular angular distribution is: :

$$
N(\Omega) d \Omega=N f(\Omega) d \Omega
$$

where $d \Omega$ is the solid angle increment, $d \Omega=\sin \theta \mathrm{d} \theta \mathrm{d} \phi$, and $f(\Omega)$ is molecular orientation distribution function, depending on the half aperture of a fluorophore cone $\Psi$, with a variable thickness $\Delta \Psi$ :

$$
f(\theta, \phi)=\frac{e^{\left(-\frac{\phi-\Psi}{\Delta \Psi}\right)^{2}}}{2 \pi \sqrt{\pi} \sin (\Psi) \Delta \Psi} \sin \theta d \theta d \phi
$$

To simplify the model, it was normalized, so the molecule density factor $N$ was omitted, and an assumption was made that transition dipole moments are collinear: $\vec{\mu}_{a b s}=\vec{\mu}_{e m} \sim \vec{\mu}$. Then, $\vec{\mu}$ could be described using the polar and azimuthal angles $\theta$ and $\phi$ :

$$
\left[\begin{array}{l}
\mu_{X}(\theta, \phi) \\
\mu_{Y}(\theta, \phi) \\
\mu_{Z}(\theta, \phi)
\end{array}\right]=\left[\begin{array}{c}
\sin \theta \cos \phi \\
\sin \theta \sin \phi \\
\cos \theta
\end{array}\right]
$$

The incident field $\vec{E}_{\omega}$ is simplified by using a planar wave function propagating in the $z$ direction. After entering the microscope body it is reflected by the dichroic mirror and depolarized according to the model:

$$
\vec{E}_{\omega}(\Phi, \delta, \varepsilon, \omega t)=\frac{E}{\sqrt{1+(1-\delta)^{2}}}\left[\begin{array}{c}
\cos \Phi \cos (\omega t) \\
(1-\delta) \sin \Phi \cos (\omega t+\varepsilon) \\
0
\end{array}\right]
$$

where $\mathrm{E}$ is the amplitude of the incident field and $\Phi$ defines the incident polarization angle in the $x y$ reference plane. $\delta$ and $\varepsilon$ are two parameters accounting for the variation of the electric field by polarization mixing and depolarization effects caused by the setup. $\delta$ represents the difference between the reflectivity of $p\left(r_{p}\right)$ and $s\left(r_{s}\right)$ polarized light from dichroic mirror and $\varepsilon$ is the ellipticity induced by it on the reflected beam.

The probability of 2PA can be expressed as tensorial product of absorption dipoles and excitation fields. Further, contraction of tensors allows to formulate it using scalar values:

$$
\begin{gathered}
P_{a b s 2}=\mu_{a b s, X}^{4} f E_{X}^{4}+\mu_{a b s, Y}^{4} f E_{Y}^{4}+6 \mu_{a b s, X}^{2} \mu_{a b s, Y}^{2} f E_{X}^{2} E_{Y}^{2}+4 \mu_{a b s, X}^{3} \mu_{a b s, Y} f E_{X}^{3} E_{Y} \\
+4 \mu_{a b s, X} \mu_{a b s, Y}^{3} f E_{X} E_{Y}^{3}
\end{gathered}
$$

Coefficients preceding the field amplitudes could be replaced by $a_{I J K L}$ (where $I, J, K, L \in[X$ $, Y])$, which are the functions of $(\theta, \phi)$ :

$$
P_{a b s 2}(\theta, \phi)=\sum_{I, J, K, L=X, Y} a_{I J K L}(\theta, \phi) E_{I} E_{J} E_{K} E_{L}
$$

While describing the probability of emission, one must take into consideration a modification of the emitted light polarization caused by high numerical aperture objective and strong focusing. The influence of high numerical aperture objective on the fluorescence 
polarization detection was described previously by Le Floc'h et al. ${ }^{2}$ and, when integrated, shortens to:

$$
\begin{aligned}
& J_{x}(\theta, \phi)=\mu_{X}^{2}(\theta, \phi) \cdot K_{1}+\mu_{Y}^{2}(\theta, \phi) \cdot K_{2}+\mu_{Z}^{2}(\theta, \phi) \cdot K_{3} \\
& J_{Y}(\theta, \phi)=\mu_{X}^{2}(\theta, \phi) \cdot K_{2}+\mu_{Y}^{2}(\theta, \phi) \cdot K_{1}+\mu_{Z}^{2}(\theta, \phi) \cdot K_{3}
\end{aligned}
$$

Where $\mathrm{K}$ factors are calculated based on the objective properties.

In the end, a factor $f_{\text {WWIJKL }}$ was defined. It combines the absorption and emission polarization properties of a molecule with the assumption of parallel dipole moments $\left(\vec{\mu}_{a b s}=\right.$ $\left.\vec{\mu}_{e m} \sim \vec{\mu}\right)$ :

$$
f_{\mathrm{WWIJKL}}(\theta, \phi)=J_{W}(\theta, \phi) \times a_{I J K L}(\theta, \phi)
$$

Where $\mathrm{W}$ denotes the polarization of the detection $(\mathrm{W} \epsilon[\mathrm{X}, \mathrm{Y}, \mathrm{Z}])$ and $\mathrm{I}, \mathrm{J}, \mathrm{K}, \mathrm{L}$ correspond to the incident polarization (I, J, K, $\mathrm{L} \in[\mathrm{X}, \mathrm{Y}]$ ). Then, the total intensity of two-photon

fluorescence excited in the distribution of molecules could be written as:

$$
I_{t}^{2 P E F}(\Phi, \delta, \varepsilon)=\int_{0}^{\pi} \int_{0}^{2 \pi} f_{\mathrm{WWIKL}}(\theta, \phi) E_{I} E_{J} E_{K} E_{L}(\Phi, \delta, \varepsilon) \sin \theta d \theta d \phi
$$

\section{Data analysis:}

To calculate the average $\Psi$ of both types of spherulites 16 data points were used: per 2 spots consisting of ordered fibrils, mostly along the $\mathrm{X}$ and $\mathrm{Y}$ axis, were chosen for 4 spherulites of different sizes for spherulites with ThT and label-free. To perform the calculations a Python

a)

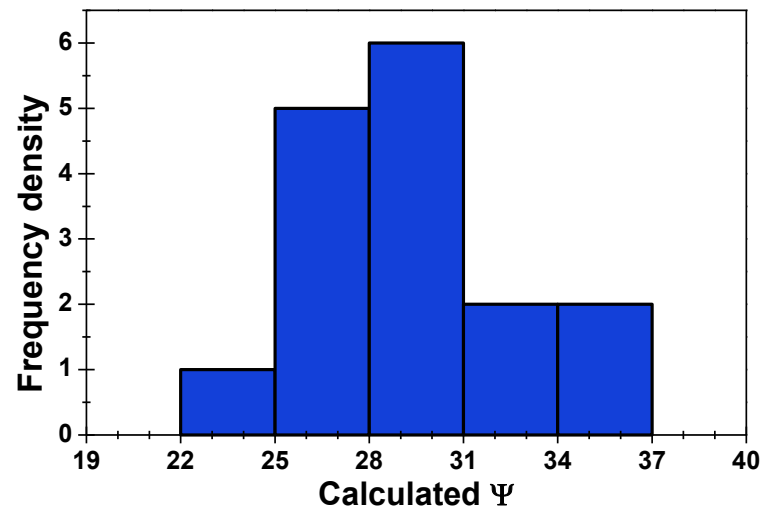

b) ThT $\Psi$ distribution

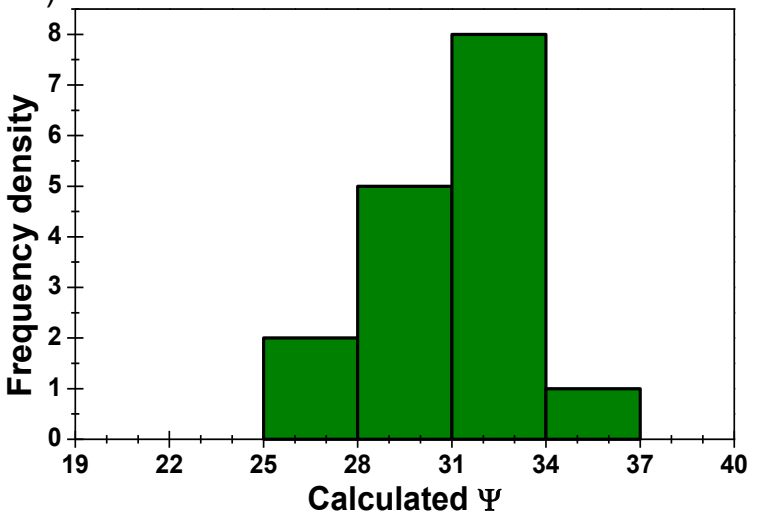

Figure S4. Distribution of calculated $\Psi$ for (a) label-free spherulites and (b) spherulites incubated with Thioflavin T.

script based on the theory given in Section 3 was used. During the calculations, the long fibril axis $(\Phi)$ was fixed at 0 and 90 degrees, depending on the chosen location. The statistical distribution of $\Psi$ obtained from the fitting of ThT polar graphs had a gaussian character with the center at $30-31^{\circ}$ while the statistical distribution of $\Psi$ from autofluorescence was more evenly distributed (Figure S4). $\Delta \Psi$ was determined during the fitting with $\Psi$ fixed at calculated averaged values equal to $30^{\circ}$ and $29^{\circ}$, for ThT and autofluorescence, respectively. The numerical calculations were done in Spyder Python 3 IDE and based on the functions available 
in libraries NumPy and SciPy. The fitting script consisted of loops checking all the searched parameters for the best, scientifically allowed, fit, basing on the QoF. During $\Psi$ and $\Delta \Psi$ fitting process the loop step was set to $2^{\circ}$. To indicate the quality of fitting the $\mathrm{R}^{2}$ factor of $\mathrm{P}=\mathrm{P}_{\mathrm{X}}+\mathrm{P}_{Y}$ was calculated, using a built-in function in Python NumPy library. $\mathrm{R}^{2}$ was in the range from 0.81 to 0.89 for spherulites stained using Thioflavin $\mathrm{T}$ and from 0.71 to 0.81 for those labelfree. Calculated $\mathrm{R}^{2}, \Psi, \Delta \Psi$ along with used $\Phi$ are attached to the corresponding polar graphs in Figure 3 and Figure S8.

\section{Atomic Force Microscopy imaging of separated insulin fibrils:}

For the AFM imaging bovine insulin, purchased from Sigma Aldrich, was dissolved in $\mathrm{HCl}$ solution $(\mathrm{pH}=1.4)$ with varying salt concentrations, namely 0,50 and $100 \mathrm{mM} \mathrm{NaCl}$. The concentration of insulin was $\mathrm{c}=2.0 \mathrm{mg} / \mathrm{mL}$. The samples followed incubation at $45{ }^{\circ} \mathrm{C}$ and agitation at $700 \mathrm{rpm}$ for $27 \mathrm{~h}$. AFM imaging was performed using a Veeco Dimension V atomic force microscope in the tapping mode. The measurements were conducted after the incubation period. The samples were diluted to $\mathrm{c}=0.02 \mathrm{mg} / \mathrm{mL}$. After 5 minutes of adsorption period, the samples were rinsed from mica layer with Milli-Q water and subsequently dried. AFM analysis was performed using Nanoscope Software 7.30. The width and height profiles were calculated according to 70 profiles. The pitch distance was measured for 10 profiles.

Performed AFM analysis revealed that ionic strength influences the morphology of as-obtained amyloid fibrils. According to the presented results (Figures S5-S6, Table S1), fibrils incubated in the absence of any salt were characterized by a mean width of $22.4 \pm 5.3 \mathrm{~nm}$. Fibrils incubated at $50 \mathrm{mM}$ were thinner, as their mean width reached a value of $18.2 \pm 4.0 \mathrm{~nm}$. At $100 \mathrm{mM} \mathrm{NaCl}$, the mean width of fibrils amounted to $19.0 \pm 5.3 \mathrm{~nm}$. Our results suggest that with the increase of ionic strength, the height of fibrils tends to increase, since at $0 \mathrm{mM} \mathrm{NaCl}$ the mean height equals $3.8 \pm 1.5 \mathrm{~nm}$, at $50 \mathrm{mM} \mathrm{NaCl} 5.6 \pm 1.7 \mathrm{~nm}$ and at $100 \mathrm{mM} \mathrm{NaCl} 6.0 \pm 1.9 \mathrm{~nm}$. The most significant changes concern twist periodicity among amyloid fibrils. We have observed that with the increase of ionic strength, the twist pitch distance tends to increase. The problem can arise from the fact that fibrils grown at such high ionic salt were much shorter than fibrils incubated at 50 or $0 \mathrm{mM} \mathrm{NaCl}$. In the absence of any salt, the twist pitch length was found within the range $85-134 \mathrm{~nm}$. At $50 \mathrm{mM} \mathrm{NaCl}$, fibrils had a twist pitch distance ranging from 121 to $202 \mathrm{~nm}$. However, at $100 \mathrm{mM} \mathrm{NaCl}$, it was impossible to measure their periodicity, since the fibrils formed untwisted morphology. The obtained results evidence that the presence of salt causes screening of electrostatic interactions in the samples, which results in ionic strengthdependent periodic twist among fibrils. 
a)

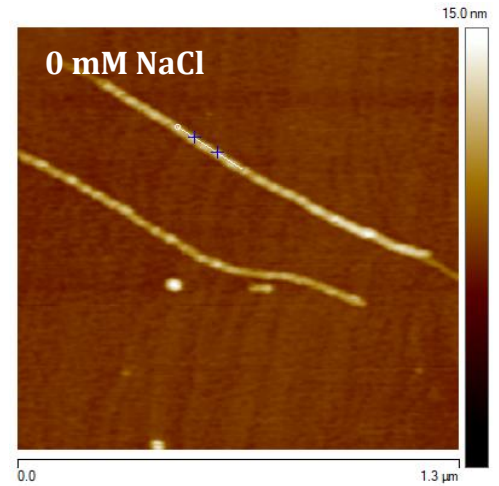

c)

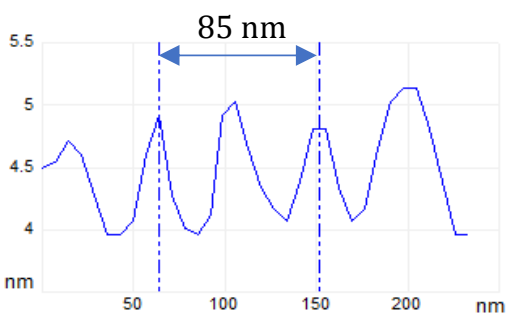

b)

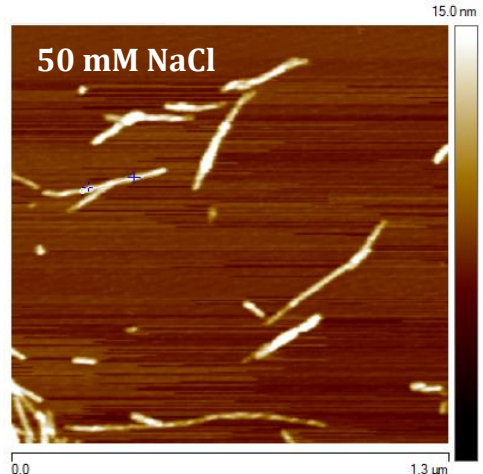

d)

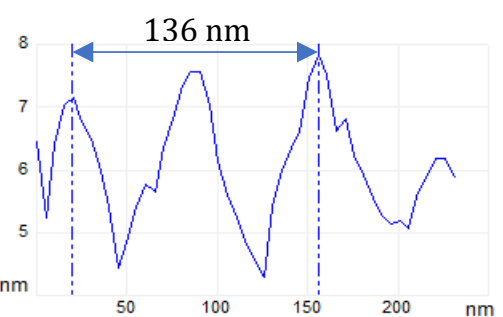

Figure S5. AFM images $(a, b)$ and the corresponding height profiles $(c, d)$ presenting twist pitch distance over highlighted fibrils' fragment incubated at $0(a, c)$ and $50 \mathrm{mM} \mathrm{NaCl}(b, d)$. Twist pitch distance values are designated with blue vertical lines $(c, d)$. 
a)

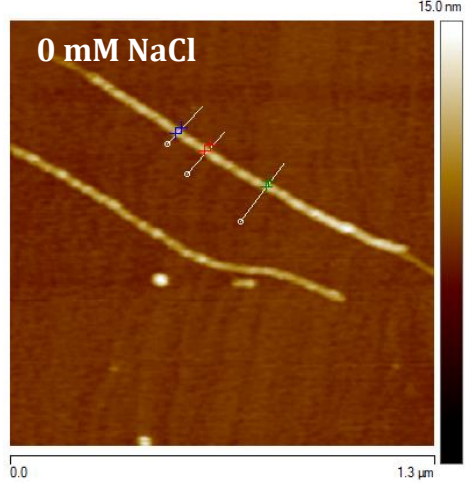

c)

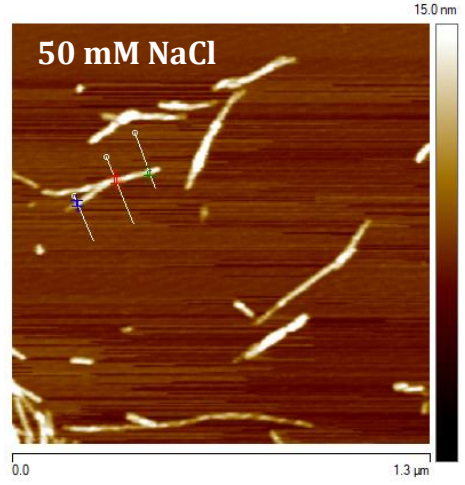

e)

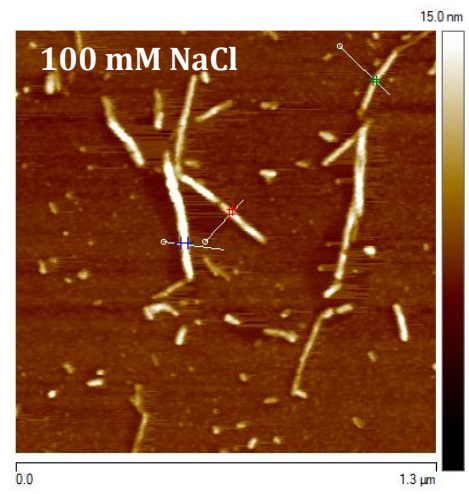

b)

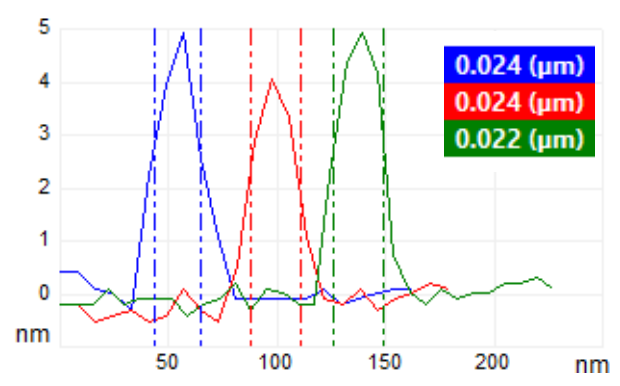

d)

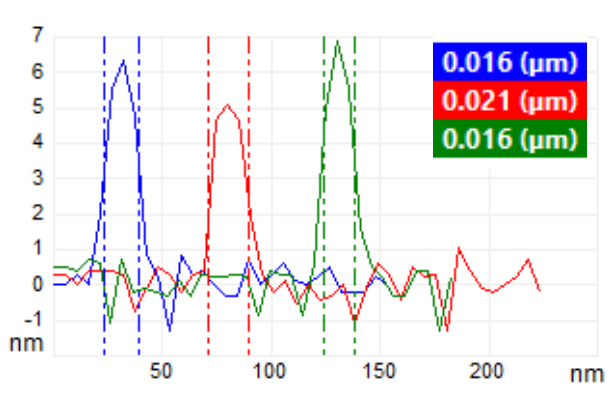

f)

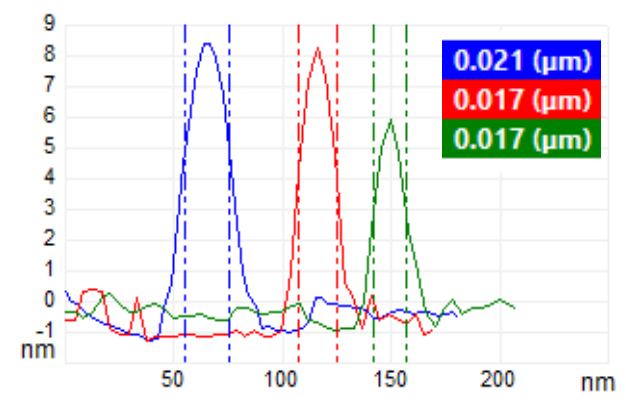

Figure S6. AFM images $(a, c, e)$ and the corresponding cross-sectional images $(b, d, f)$ demonstrating width and height distribution of analyzed amyloid fibrils incubated at $O(a, b)$, $50(c, d)$ and $100 \mathrm{mM} \mathrm{NaCl}(e, f)$. The blue, red and green rectangulars on the cross-sectional images demonstrate examined width values. 
Table S1. Mean width, height and twist pitch distance of amyloid fibrils obtained after incubation at varying $\mathrm{NaCl}$ concentrations. Mean width and height values were estimated according to measured 70 profiles. Twist pitch length is given as the range seen in 10 measured profiles.

\begin{tabular}{|l|c|c|c|}
\hline \multicolumn{1}{|c|}{ Sample } & Mean width (nm) & Mean height (nm) & Twist pitch length (nm) \\
\hline $0 \mathrm{mM} \mathrm{NaCl}$ & $22.4 \pm 5.3$ & $3.8 \pm 1.5$ & $85-134$ \\
\hline $50 \mathrm{mM} \mathrm{NaCl}$ & $18.2 \pm 4.0$ & $5.6 \pm 1.7$ & $121-202$ \\
\hline $100 \mathrm{mM} \mathrm{NaCl}$ & $19.0 \pm 5.3$ & $6.0 \pm 1.9$ & - \\
\hline
\end{tabular}

6. Polarization-sensitive two-photon microscopy imaging of ThT-labeled spherulites:
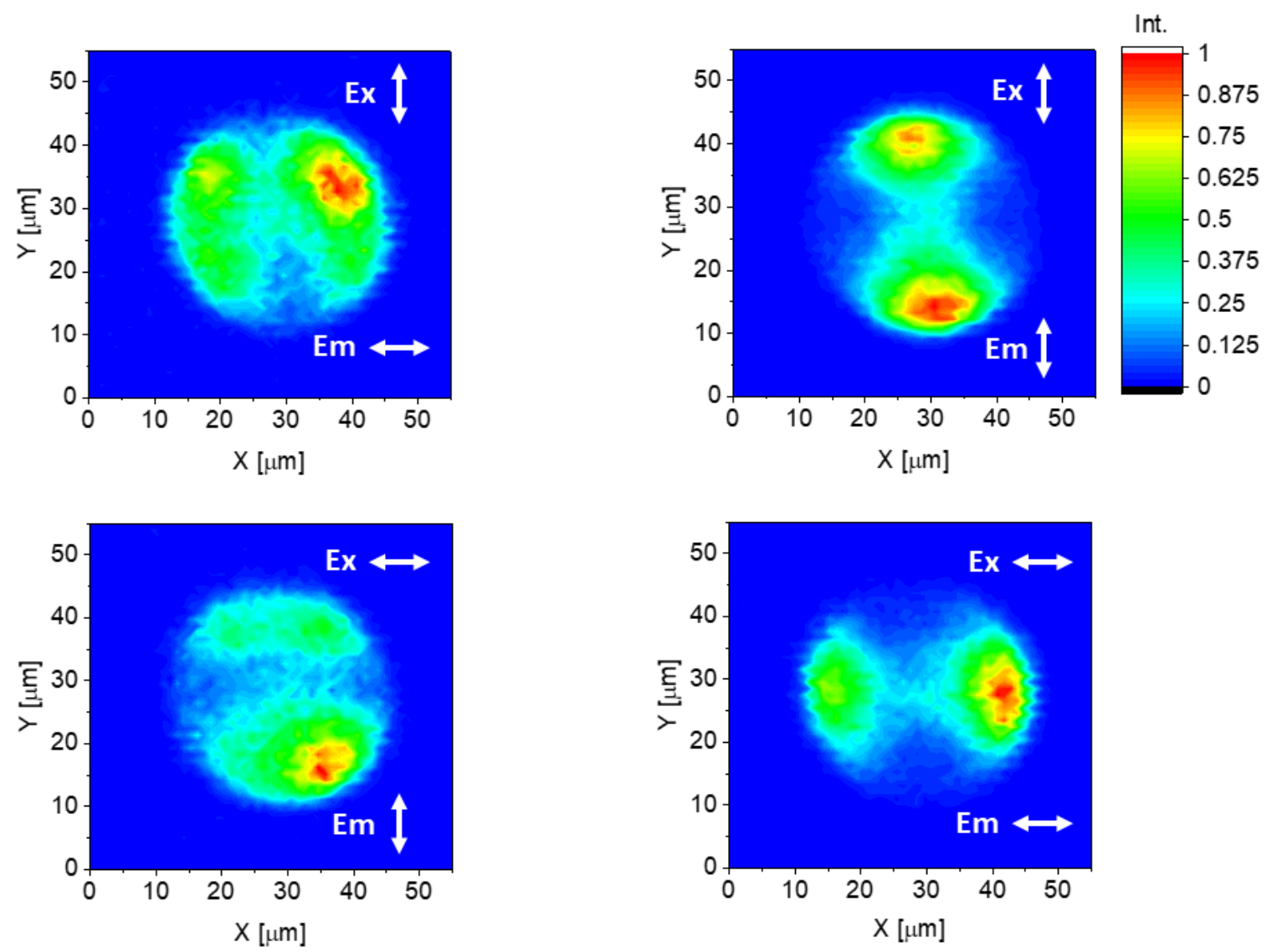

Figure S7. 2PEF intensity raster scans of a ThT-labeled insulin spherulite. Polarization of excitation beam (Ex) and emission (Em) are denoted with white arrows. The excitation wavelength was set to $810 \mathrm{~nm}$. The same spherulite imaged under polarized light microscope with crossed polarizers is available in the Supporting Information (Figure S3). 

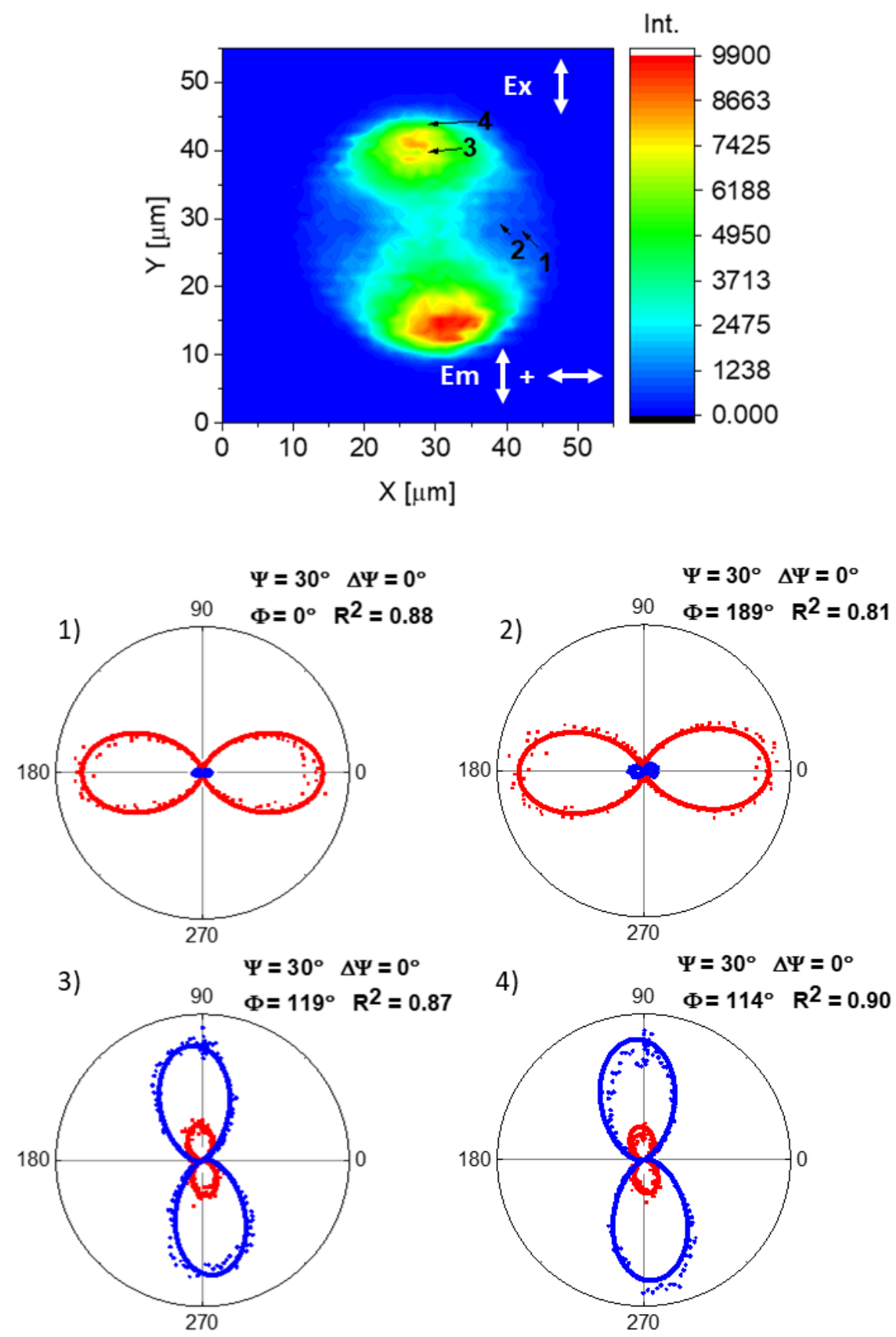

Figure S8. XY raster scan of ThT-labeled spherulite excited with vertically polarized light and polar graphs from spots denoted on the scan. Experimental data are presented as dots $\left(I_{x}-\right.$ red, $I_{y}$ - blue), and solid lines presents the fitting of the angular dependence of two-photon excited emission. Polarization of excitation light (Ex) and emission (Em) are denoted with white arrows. The excitation wavelength was set to $810 \mathrm{~nm}$.

\section{References:}

1. Brasselet, S. Polarization-resolved nonlinear microscopy: application to structural molecular and biological imaging. Adv. Opt. Photon. 2011, 3 (3), 205.

2. Le Floc'h, V.; Brasselet, S.; Roch, J.-F.; Zyss, J. Monitoring of Orientation in Molecular Ensembles by Polarization Sensitive Nonlinear Microscopy. The Journal of Physical Chemistry B 2003, 107 (45), 12403-12410. 